\title{
Leistungsmodellierung als Voraussetzung für Produktionsplanung und -steuerung (PPS) für die Bereitstellung von IT-Services
}

\author{
Darstellung anhand des Fallbeispiels der Global \\ Delivery Unit SAP Services der T-Systems Enterprise \\ Services GmbH
}

\author{
Alexander Vogedes, Nico Ebert, Henrik Brocke, Falk Uebernickel, \\ Walter Brenner
}

Universität St. Gallen, Institut für Wirtschaftsinformatik, Müller-Friedberg-Straße 8, 9000 St. Gallen, Schweiz, vorname.nachname@unisg.ch

\begin{abstract}
Steigender Kostendruck und zunehmende Dienstleistungsorientierung erfordern ein Umdenken beim Einsatz von Methoden zum Management der Bereitstellung von IT-Services. Wurden in der Vergangenheit primär Projektmanagementmethoden genutzt, rücken mit zunehmender IT-Service-Standardisierung Methoden aus der Sachgüterfertigung in den Mittelpunkt. In diesem Beitrag wird dargestellt, welche Möglichkeiten und Grenzen industrielle Produktionsplanungsund -steuerungssysteme für die Bereitstellung von IT-Services am konkreten Fallbeispiel der „Global Delivery Unit SAP Services“ der T-Systems Enterprise Services $\mathrm{GmbH}$ aufweisen.
\end{abstract}

\section{Ausgangssituation und Ziel des Beitrags}

Steigender Optimierungs- und Kostendruck führt bei IT-Organisationen zum Wandel vom projektorientierten zum ,industrialisierten“ IT-Service-Management (CapGemini, 2008, S. 145). Die Adaption etablierter Konzepte aus der Industrie resultiert erstens in kundenorientierten, standardisierten IT-Services (Grawe and Fähnrich, 2008). Diese bilden dabei zunehmend integrierte IT-Service-Bündel zur Unterstützung von Kundenprozessen anstelle systemnaher IT-Services wie Serveroder Netzbetrieb ab (Zarnekow, 2007). Zweitens betrifft diese „Industrialisierung“ die Auftragsabwicklung und dabei insbesondere die IT-Produktion, welche Betrieb, Wartung und Support von Betriebsmitteln zur effizienten Erfüllung solcher komplexen kundenorientierten Leistungszusagen gewährleistet.

Bei der Umsetzung dieser Industrialisierung werden jedoch Herausforderungen deutlich. Zwar haben mittlerweile viele IT-Organisationen ihr IT-Service-Portfolio 
in Katalogen beschrieben, eine standardisierte Beauftragung und Auftragsabwicklung dieser IT-Services erfolgt jedoch meist nicht. Neben einer elektronischen Auftragsannahme fehlt es zur effizienten, qualitativ hochwertigen Produktion solcher komplexen, integrierten IT-Service-Bündel insbesondere an einer computergestützten Produktionsplanung und -steuerung der Erbringung der IT-Services. Bisher werden ITIL-konforme Service- bzw. Workflow-Managementsysteme eingesetzt, die planerische Aspekte im Hinblick auf den effizienten und effektiven Ressourceneinsatz vernachlässigen.

Experten gehen entsprechend davon aus, dass ein Großteil der Kapazitäten in der IT-Produktion nur zu einem geringen Grad ausgelastet sind (Zarnekow, 2007, McKinsey \& Company, 2008). Das entstehende Optimierungspotenzial wird unter der Berücksichtigung deutlich, dass etwa 55\%-75\% des IT-Budgets in Unternehmen der laufenden Produktion zugerechnet werden kann (Gartner, 2008, McKinsey \& Company, 2008). Überdies verspricht eine optimierte Bereitstellung eine wesentlich effizientere und schnellere Auftragsabwicklung eingehender Kundenanforderungen. Diese Ziele verfolgend, schlägt der vorliegende Beitrag die Konzeptübertragung der Produktionsplanung und -steuerung (PPS) auf die ITProduktion vor und setzt dabei den Fokus auf die Bereitstellung. Für die dafür benötigten Daten wird ein Modellierungsvorschlag vorgestellt, der anhand eines realen Fallbeispiels (Ebert, 2009, S. 47 ff., Ebert and Vogedes, 2008) die Anwendung eines PPS-Systems innerhalb einer IT-Organisation veranschaulicht.

\section{Forschungsvorgehen}

Unter Berücksichtigung IT-spezifischer Besonderheiten wird im Folgenden der Einsatz der Produktionsplanung und -steuerung im IT-Service-Management für die Bereitstellung von IT-Services vorgeschlagen. Der vorgeschlagene Ansatz basiert auf den Grundlagen und Anforderungen der Bereitstellung von IT-Services von Ebert et al. (Ebert et al., 2009) und prüft diese anhand des konkreten Kontextes eines Fallbeispiels des Bereichs „Global Delivery Unit SAP Services“ der TSystems Enterprise Services GmbH (Ebert, 2009, S. 47 ff.). Zur Überprüfung der Nützlichkeit dieses Ansatzes wird dabei jeweils die Verwendung der PPS in der Bereitstellung anhand des Bereichs „Global Delivery Unit SAP Services“ der TSystems Enterprise Services GmbH beispielhaft diskutiert (Ebert, 2009, S. 47 ff.).

Der betrachtete Bereich umfasst etwa 800 Mitarbeiter und betreut knapp 2700 SAP-Installationen (Ebert, 2009, S. 47 ff.). Im Mittelpunkt der Produktion stehen die „Dynamic Services for SAP Solutions“. Dabei handelt es sich um den Betrieb von SAP-Systemen auf einer flexiblen Infrastruktur-Plattform (,AppComPlattform"), die sich aus standardisierten und virtualisierten Infrastrukturkomponenten für Storage, Rechenzentrumsnetze und Server sowie zentralen Managementmethoden und -verfahren zusammensetzt. Diese Plattform ermöglicht den Betrieb von SAP, Non-SAP und Web-Anwendungen sowie Datenbanken. Eine solches Betriebskonzept wird als Adaptive Computing bezeichnet, dabei steht der 
Betrieb von Anwendungen im Vordergrund an deren dynamische Anforderungen die Ressourcen der darunterliegenden IT-Infrastruktur anzupassen sind (Osterburg et al., 2009). Unter dem Stichwort Cloud Computing wird u.a. ein automatisiertes Adaptive Computing verstanden (Osterburg et al., 2009).

\section{PPS-Systeme zur Bereitstellung von IT-Services}

Bei einem PPS-System handelt es sich um ein Softwaresystem, das zur Unterstützung der operativen Planung und Steuerung des Fertigungsgeschehens in einem Industriebetrieb eingesetzt wird (Kurbel, 2005, S. 1). Das MRP-II-Konzept verfolgt den Ansatz einer hierarchischen Sukzessivplanung und bildet die Grundlage für die meisten PPS-Systeme (Kurbel, 2005, S. 38). Trotz der Weiterentwicklungen der PPS-Systeme zu heutigen Enterprise Resource Planning-Systemen sind deren Kernfunktionalitäten im Bereich Fertigung nach wie vor identisch (Mertens, 2007, S. 117ff.).

Während in der Vergangenheit die Verwendung von PPS im IT-Betrieb (Lebrecht, 1991) betrachtet wurde, steht im Mittelpunkt der nachfolgenden Betrachtungen die Nutzung von PPS für die Bereitstellung. Im Lebenszyklus eines ITServices erfolgt die Bereitstellung mit der Beauftragung eines zuvor spezifizierten IT-Services und ist dem IT-Betrieb vorgelagert. Die Bereitstellungsphase beinhaltet die Installation und Konfiguration der erforderlichen Betriebsmittel und erfordert in der Regel die Integration des Kunden, der Daten wie Benutzerkennungen, technische Netzwerk- und Systemschnittstellen und Anwendungsdaten bereitstellen muss. Im anschließenden IT-Betrieb wird der IT-Service dann durch den Benutzer verwendet und die Wartung der Systeme erforderlich. Der Lebenszyklus endet mit der Deinstallation des IT-Services und der Freigabe entsprechender Betriebsmittel (Garschhammer et al., 2001a, Zarnekow, 2007, S. 112).

Aus produktionswirtschaftlicher Sicht handelt es sich bei der Bereitstellung um ein System, in dem Produktionsfaktoren (Input) in einer Faktorkombination (Throughput) zu Leistungen (Output) kombiniert werden (Zäpfel, 1982, S.2). Produktionsfaktoren sind dabei primär Werkstoffe wie Server, Datenspeicher, Netzwerke und Anwendungen. Bei den Potenzialfaktoren handelt es sich um Betriebsmitteltypen wie Bereitstellungssysteme und um menschliche Arbeitsleistung (Zarnekow, 2007, S. 88). Diese Produktionsfaktoren werden in manuellen und/oder automatischen Prozessen zu produktiven Betriebsmitteln kombiniert. Im Gegensatz zur klassischen Fertigung sind dabei insbesondere drei Hauptunterschiede zu beachten:

- IT-Service vs. Service-Implementierung: Ein zentrales Prinzip der ServiceOrientierung ist die Trennung zwischen IT-Service und dessen ServiceImplementierung (Garschhammer et al., 2001b). Ein IT-Service ist als Spezifikation funktionaler und nicht-funktionaler Anforderungen zu verstehen. Im Unterschied dazu stellt die Service-Implementierung, d.h. ein produktives Be- 
triebsmittel, die Lösung zur Umsetzung dieser Anforderungen dar. Durch die Trennung hat der Provider die Möglichkeit, einen IT-Service nach eigenem Ermessen zu implementieren - solange die Implementierung den Anforderungen des IT-Services genügt. Zwischen IT-Service und Implementierung besteht eine n:m-Beziehung. Als Konsequenz aus der Trennung muss der Bedarf für Produktionsfaktoren der Implementierung aus dem Bedarf für IT-Services abgeleitet werden, da letztere den primären Output der IT-Organisation darstellen. Begrifflich wird die Implementierung des IT-Services folgend Betriebsmitteltyp genannt, damit dem Leser die gedankliche Verbindung in die reale Welt erleichtert wird. Ein Betriebsmitteltyp wird durch den Vorgang der Bereitstellung (Vorgang der Instanziierung) zu einet (konkreten) Betriebsmittelinstanz (siehe Abb. 1).

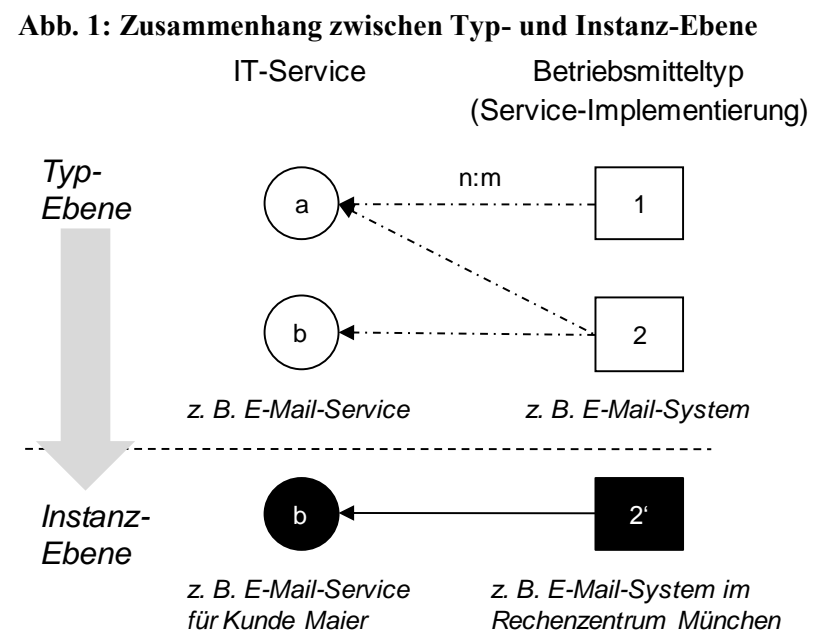

- Zeitraumbezogene Instanzen: Da die Beauftragung eines IT-Services zeitraumbezogen erfolgt, existieren IT-Service-Instanzen eines IT-Services und Betriebsmittel eines Betriebsmitteltyps. In der Praxis werden die Bestände der Instanzen in einer Configuration Management Database (CMDB) dokumentiert (OGC, 2007). Folglich kann die Bereitstellung neuer IT-Services respektive erforderlicher Betriebsmittel nicht ,auf der grünen Wiese“ stattfinden, sondern unter Berücksichtigung vorhandener Instanzen.

- Externe Faktoren: Bei externen Faktoren handelt es sich um Personen oder Objekte, die nicht der Dispositionsgewalt der IT-Organisation unterliegen, sondern vom Kunden festgelegt werden. Z. B. muss der Kunde der ITOrganisation unter Umständen während der Bereitstellung Migrationsdaten bereitstellen. Da die externen Faktoren durch den Provider schwer zu beeinflussen sind, müssen potenziell verbundene Unsicherheiten berücksichtigt werden (z. B. als Planannahmen) (Corsten and Gössinger, 2007, S. 119). Weiterhin gilt 
es deshalb, die Integration des Kunden zur Aufnahme der benötigten Daten weitestmöglich standardisiert und zum Zeitpunkt der Beauftragung des ITServices umzusetzen.

Unter Berücksichtigung dieser IT-spezifischen Besonderheiten wird im Folgenden der Einsatz der Produktionsplanung und -steuerung im IT-ServiceManagement zur Bereitstellung vorgestellt, indem erstens auf die Daten und zweitens auf die Funktionen der PPS näher eingegangen wird.

\section{Daten der PPS zur Bereitstellung von IT-Services}

Die wichtigsten Daten der PPS sind zum einen die auftragsrelevanten Daten seitens des Kunden und zum anderen die korrespondierenden Daten über Teile, Erzeugnisstrukturen, Arbeitspläne, Betriebsmitteltypen und Arbeitsplätze zur Produktion dieser Aufträge. Auftragsdaten werden bei der Beauftragung elektronisch unterstützt abgefragt, um eine integrierte und vollständige Datenbasis für die PPS sicherzustellen. Die Teildaten werden in einem Teilestamm hinterlegt, der relevante physikalische und betriebswirtschaftliche Daten für ein Teil enthält. Zur Repräsentation der Erzeugnisstrukturen werden Stücklisten genutzt, die Attribute wie Materialnummer, Bezeichnung oder Mengenkoeffizienten besitzen (Kurbel, 2005, S. 66). Die Arbeitspläne enthalten Arbeitsgänge, die zur Herstellung eines Teils erforderlich sind. Zwischen den Arbeitsgängen eines Arbeitsplans bestehen Reihenfolgebeziehungen, d.h. ein Arbeitsgang kann über bestimmte vorgelagerte und nachgelagerte Arbeitsgänge verfügen. Jeder Arbeitsgang verfügt über eine Zuordnung zu einem Arbeitsplatz, der diesen Arbeitsgang ausführen kann und in Anspruch genommen wird (Kurbel, 2005, S. 83ff.).

\subsection{Teilestamm und Stücklisten}

Die Trennung zwischen IT-Service und Betriebsmittel führt zu zwei Teilestämmen. Im IT-Service-Stamm werden die IT-Services und im Betriebsmittelstamm die Betriebsmitteltypen spezifiziert. Analog zum Teil in der Industrie können sich sowohl IT-Services als auch Betriebsmittel aus Komponenten zusammensetzen, die in den jeweiligen Stämmen enthalten sind. Betriebsmittelkomponenten, die der Kunde beistellt, müssen besonders gekennzeichnet werden. Zwischen den Elementen beider Teilestämme existiert eine Zuordnungsvorschrift, die mögliche Betriebsmittel für IT-Services definiert (siehe LeistungBetriebsmittel-Zuordnung in Abb. 2). Diese Zuordnung legt den Bedarf einer ITLeistung (Unterelement eines IT-Services) an Betriebsmitteltypen fest, wie in Abb. 1 durch einen Pfeil angedeutet. Diese Information wird für die Bereitstellung 
der Betriebsmittelinstanz zur Erbringung des IT-Services (Instanziierung des Betriebsmitteltyps) benötigt.

Die IT-Services und Betriebsmitteltypen verfügen über Stücklisten, deren Beziehungen jedoch unterschiedlich interpretiert werden. Die Beziehungen in den Stücklisten von Betriebsmitteltypen entsprechen denen der klassischen Fertigung. In dieser wird die Beziehung zwischen untergeordneten und übergeordneten Komponenten als ,geht ein in“ interpretiert. Im Unterschied dazu handelt es sich bei IT-Services um IT-Service-Bündel, die nach Kundenbedürfnissen zusammengestellt werden. Daher ist die Beziehung zwischen untergeordneten und übergeordneten Komponenten als „,ist Bestandteil von“ zu interpretieren. Das IT-ServiceBündel ist in Abb. 2 als IT-Service und IT-Leistung dargestellt.

Abb. 2. ER-Diagramm zu IT-Service, Betriebsmittelinstanz und Betriebsmitteltyp

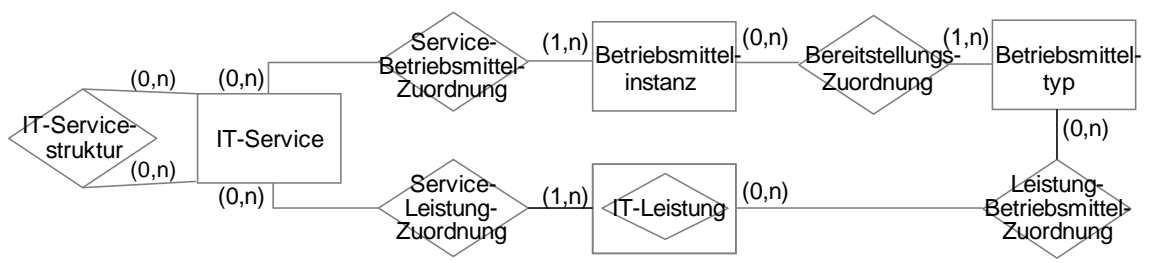

In der Fallstudie stellen Standard Service Elemente (SSE) als Leistungen des Betriebs die Schnittstelle zum Vertrieb der T-Systems dar (Ebert, 2009, S. 47 ff.). Sie können modular nach Wunsch des Kunden zusammengesetzt werden und sind einheitlich hinsichtlich Funktionalität, Quality of Service (QoS) und Kosten beschrieben. Sämtliche SSE sind in einer einheitlichen Form in einem Katalog beschrieben, der dem IT-Service-Stamm entspricht. Zur Erbringung eines SAPAnwendungsservices in einem Rechenzentrum (RZ) werden z. B. SSE zum Betrieb von SAP-Systemen, Server-Systemen, Storage-Systemen und Firewalls benötigt (siehe Tabelle 1). Bei den SSE für SAP- und Server-Systeme wird zwischen leistungsabhängigen und -unabhängigen SSE unterschieden. Während die leistungsabhängigen IT-Services von der Output-Leistung eines Systems abhängen, sichern die leistungsunabhängigen IT-Services den Basisbetrieb. 
Tabelle 1: Standard Service Elemente (SSE) für den SAPAnwendungsbetrieb

\begin{tabular}{|c|c|c|}
\hline $\begin{array}{l}\text { Standard Service } \\
\text { Element (SSE) }\end{array}$ & Einheit & Beschreibung \\
\hline SAP Only Operations Basic & \# Systeme & Betrieb eines SAP-Systems ink1. Datenbank \\
\hline SAP Only Operations Usage & \# SAPS $^{1}$ & $\begin{array}{l}\text { Betrieb einer SAP-Systemkapazität (leistungsab- } \\
\text { hängig) }\end{array}$ \\
\hline $\begin{array}{l}\text { Open Systems AppCom } \\
\text { Operations }\end{array}$ & \# Server & $\begin{array}{l}\text { Betrieb eines dedizierten oder virtuellen Servers } \\
\text { ink1. Betriebssystemlizenz (leistungsunabhängig) }\end{array}$ \\
\hline $\begin{array}{l}\text { Open Systems Hardware Slices } \\
\text { Operations }\end{array}$ & \# Slices ${ }^{2}$ & Betrieb eines Slices (leistungsabhängig) \\
\hline $\begin{array}{l}\text { Backup Integrated Storage } \\
\text { Operations }\end{array}$ & \# TByte & $\begin{array}{l}\text { Bereitstellung und Betrieb von täglich gesichertem } \\
\text { Storage eines Dateiservers via Netzwerk }\end{array}$ \\
\hline Firewall Operations & \# Firewalls & Betrieb einer Firewall zwischen RZ und WAN \\
\hline
\end{tabular}

Eine vereinfachte Stücklistendarstellung eines IT-Services zum Betrieb eines SAP-Systems inkl. Storage und Anbindung an das Rechenzentrumsnetz zeigt Abb. 3. Der IT-Service setzt sich aus SSE-Modulen für den leistungsabhängigen und -unabhängigen Betrieb eines SAP-Systems zusammen. Beide Module beinhalten ihrerseits SSE für den Server-Betrieb und den SAP-Betrieb. Neben dem Betrieb für das SAP-System werden weitere SSE für den Betrieb des Storage und der Firewall im IT-Service gebündelt.

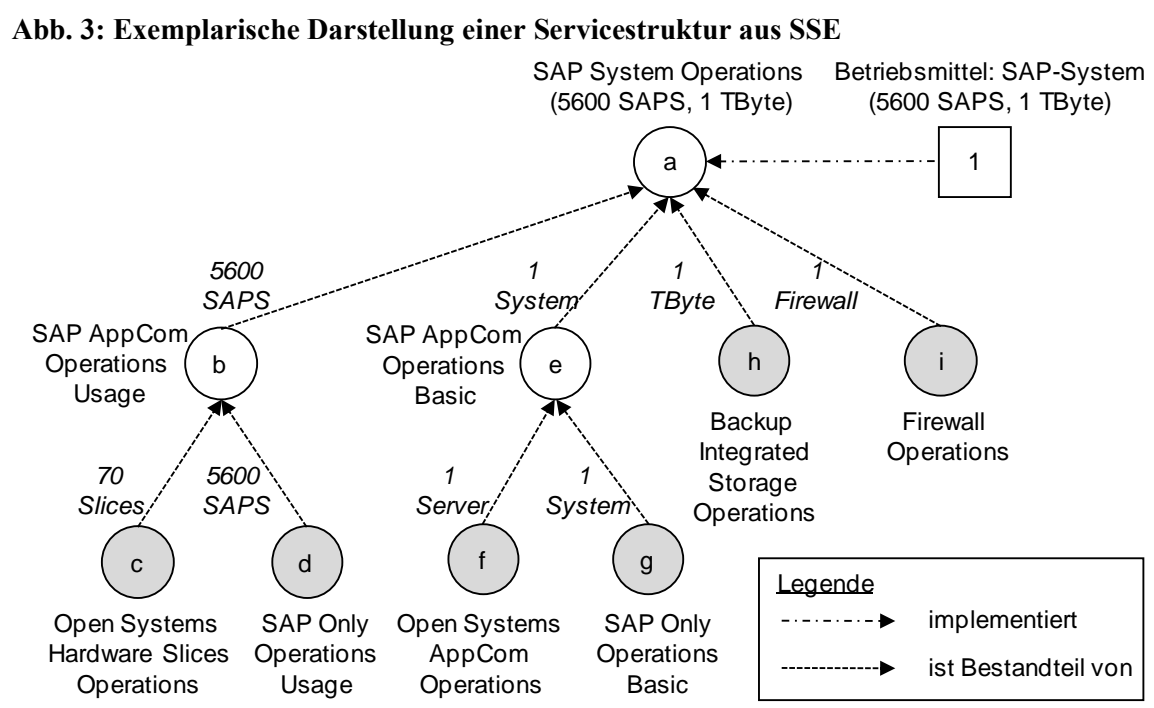

${ }^{1}$ SAP Application Performance Standard (SAPS) ist eine Einheit für die Leistungsfähigkeit eines SAP-Systems (Mißbach, 2005, S.145).

${ }^{2}$ Slice ist eine normierte Einheit für die Leistungsfähigkeit eines Servers (1 Slice $\approx 80$ SAPS). 
Die Bereitstellung des IT-Services erfolgt mittels eines SAP-Systems auf der AppCom-Plattform. Zur Bereitstellung der Betriebsmittel sind Komponenten für Rechen- und Speicherkapazitäten sowie für eine Firewall erforderlich (siehe Abb. 4). Im Rechner-Pool befinden sich standardisierte, physische Server mit integriertem Hauptspeicher, die Anwendungen wie SAP-R/3 ausführen. Der Pool setzt sich aus Servern unterschiedlicher Hardware-Typen mit unterschiedlichen Betriebssystemen (z. B. Intel-Server mit Linux) zusammen. Durch eine Virtualisierungslösung können kurzfristig virtuelle Server angelegt werden, mit denen ein physischer Server in logische Einheiten aufgeteilt wird. Die Anwendungs- bzw. Datenbankserver sind über ein Gigabit-Netz mit dem Storage-Pool verbunden, der sich aus dedizierten Storage-Rechnern zusammensetzt. Innerhalb eines Rechenzentrums sind Server und Storage eines Kunden über VLAN verbunden.

Abb. 4: Exemplarische Darstellung eines Betriebsmittels

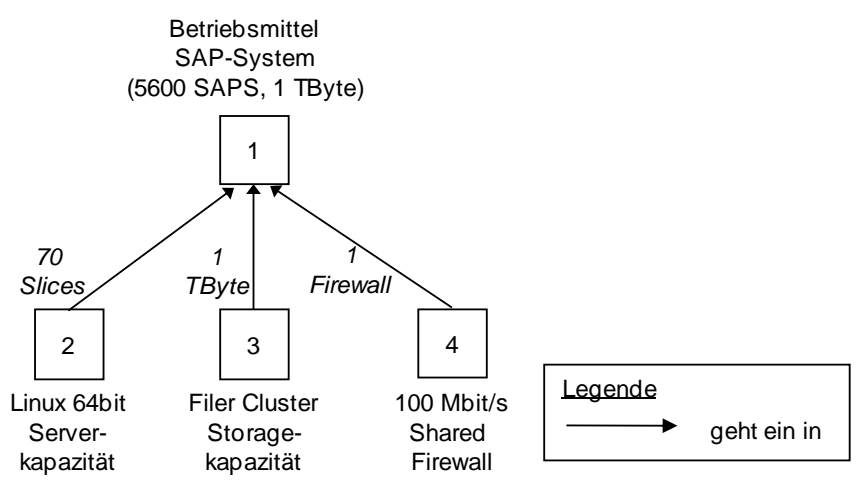

\subsection{Arbeitspläne und Arbeitsplätze}

Die Ablaufinformationen zur Bereitstellung werden als Arbeitspläne dokumentiert, die einem Betriebsmittel zugeordnet sind. Diejenigen Arbeitsgänge, die externe Faktoren wie die Benutzer integrieren, sind besonders kritisch für die Planung und müssen gekennzeichnet werden. Die Planwerte für diese Arbeitsgänge können aus Erfahrungswerten und SLA (Service Level Agreement) (z. B. „Mitwirkungspflichten des Kunden“") abgeleitet werden. Die Arbeitsplätze, welche die Arbeitspläne ausführen, sind organisatorische Einheiten mit definierten Kapazitäten, die über Personal und Systeme für die Bereitstellung verfügen.

In der Fallstudie wurde der Arbeitsplan des Bereitstellungsprozesses für ein SAP-System aufgenommen (siehe Tabelle 2) (Ebert, 2009, S. 47 ff.). Er zeigt die Arbeitsgänge, ausführende Arbeitsplätze und deren Stückzeiten pro System. Nach Abschluss des Vertrags konzipiert und dokumentiert der Global Delivery Unit SAP Services-Bereich (GDU SAP Services-Bereich) die Systemarchitektur, das 
Layout des Dateisystems und die Vernetzung im Rechenzentrum. Nachdem der Infrastructure \& Architecture Services-Bereich ein VLAN inkl. einer Firewall konfiguriert hat, stellt der Open System Services-Bereich konfigurierte Server inkl. Betriebssystem und Storage bereit. Für das lauffähige System führt danach der GDU SAP Services-Bereich eine Grundkonfiguration durch, die u.a. die Anlage des Dateisystems, die Konfiguration der IP-Adressen und AdministratorKonten sowie die Bereitstellung von Administrationswerkzeugen wie Start/StopSkripte oder FTP-Werkzeuge umfasst. Daran schließt sich die Installation des SAP-Systems an (inkl. Installationsplanung, Durchführung der Installation, Test und Inbetriebnahme). Sofern der Kunde bereits ein SAP-System genutzt hat, werden bestehende Kundendaten migriert. Die einzelnen Arbeitsgänge erfolgen in zunehmendem Maß automatisiert. Die Mitarbeiter der T-Systems werden durch Bereitstellungssysteme z. B. Serverkonfiguration oder Softwareinstallation unterstützt.

Tabelle 2: Exemplarischer Arbeitsplan zur Bereitstellung eines SAP-Systems (Stückzahlen fiktiv)

\begin{tabular}{llll}
\hline \multicolumn{4}{l}{ Bereitstellungsarbeitsplan: SAP-System bereitstellen } \\
\hline Nr. & Beschreibung & Arbeitsplatz & Stückzeit \\
\hline 01 & Systemkonzeption & GDU SAP Services & $200 \mathrm{~min}$ \\
\hline 02 & Kunden IP zuweisen & Infrast. \& Architecture Services & $15 \mathrm{~min}$ \\
\hline 03 & Firewall-Konfiguration & Infrast. \& Architecture Services & $30 \mathrm{~min}$ \\
\hline 04 & Server/Storage konfigurieren & Open System Services & $120 \mathrm{~min}$ \\
\hline 05 & SAP-Grundkonfiguration & GDU SAP Services & $180 \mathrm{~min}$ \\
\hline 06 & SAP-Installation & GDU SAP Services & $180 \mathrm{~min}$ \\
\hline
\end{tabular}

\section{Funktionen der PPS zur Bereitstellung von IT-Services}

Die klassischen Funktionen von PPS-Systemen können gemäß ihrer Fristigkeit in lang-, mittel- und kurzfristige Funktionen strukturiert werden (siehe Abb. 5). In der „Produktionsprogrammplanung“ werden die zu produzierenden Erzeugnisse festgelegt. Die Aufgabe der „Mengenplanung“ besteht in der Bestimmung der Fertigungs- bzw. Bestellaufträge für alle Komponenten nach Art und Zeit unter Beachtung des Programms. Es kann dazu eine Stücklistenauflösung genutzt werden, welche den Bedarf an Komponenten aus dem Produktionsprogramm errechnet. Die „Termin- und Kapazitätsplanung“ ermittelt anhand der Arbeitspläne die Startund Endtermine der Arbeitsgänge für die geplanten Fertigungsaufträge. Die anschließende „Auftragsveranlassung“ sorgt für die kurzfristige Durchsetzung des Produktionsprogramms. Schließlich dient die „Auftragsüberwachung“ der Erfassung und Verwaltung von Zustandsänderungen der Aufträge und Kapazitäten. Als Querschnittsfunktion ist die Datenverwaltung für die Datenhaltung und Kommu- 
nikation zwischen Produktionsplanung und Produktionssteuerung zuständig (Hackstein, 1989, S. 15).

Abb. 5: Klassische Funktionsstruktur eines PPS-Systems in Anlehnung an (Hackstein, 1989, S. 5)

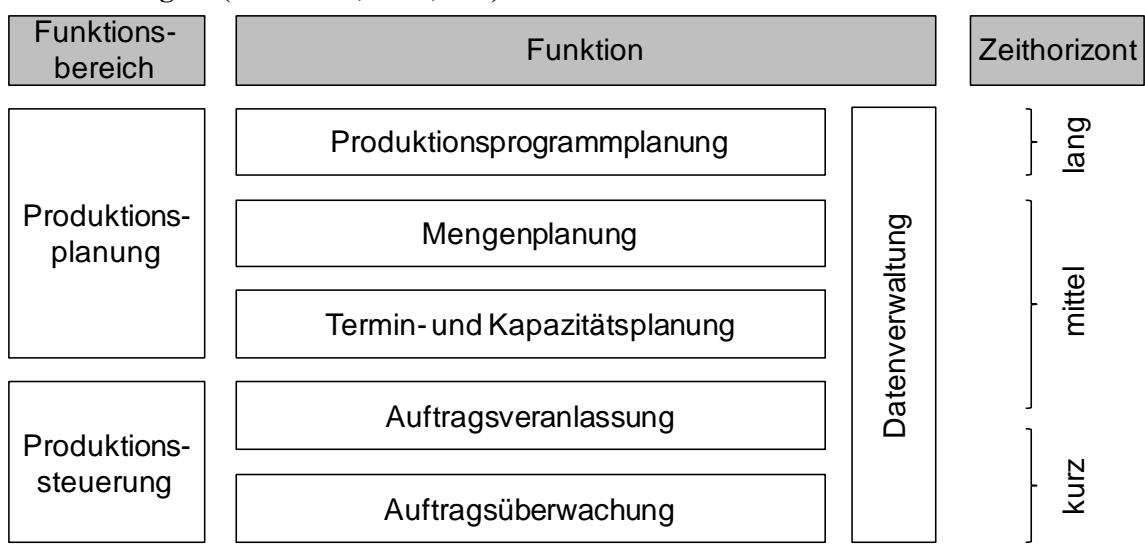

\subsection{Programmplanung}

Die Bereitstellung ist vergleichbar mit der Kundenauftragsfertigung in der Industrie. Im Gegensatz zu einer Lagerfertigung, die unabhängig vom Kundenauftrag ausgeführt wird, lösen in der Kundenauftragsfertigung konkrete Kundenaufträge die Fertigung aus (Kurbel, 2005, S. 197). Anders als in der Kundenauftragsfertigung werden IT-Services nicht für einen Zeitpunkt sondern mittels SLA für einen Zeitraum beauftragt.

Die Aufgabe der Programmplanung ist die Ermittlung des Bedarfs von ITServices auf Basis von Prognosen und Kundenverträgen unter Berücksichtigung bekannter oder erwarteter Vertragszeiträume und bestehender IT-ServiceInstanzen. Das Planungsobjekt ist im Fall eines Kundenvertrags die IT-ServiceInstanz oder andernfalls der erwartete IT-Service bzw. dessen übergeordnete ITService-Familie. Aus dem Bedarf für IT-Service-Instanzen bzw. IT-Services kann mittels der Zuordnungsvorschrift der Bedarf für Betriebsmitteltypen abgeleitet werden. Wenn Prognosen für IT-Service-Bedarfe genutzt werden, müssen Annahmen über mögliche Betriebsmitteltypen getroffen werden, sofern verschiedene Varianten von Betriebsmitteltypen zulässig sind. In diese Annahme müssen auch vertrags- oder technologiebedingte Ersetzungszyklen einfließen (z. B. Ersetzung eines Desktops alle drei Jahre). Ebenso wie bei den IT-Services erfolgt die Ermittlung des Bedarfs von Betriebsmitteltypen unter Beachtung vorhandener Instanzen. Die abgeleiteten Bedarfsinformationen aus Prognosen können im Sinne einer qua- 
si deterministischen Planung zur Disposition vertragsunspezifischer Komponenten und zur Bereitstellung erforderlicher Ressourcen genutzt werden.

Die Programmplanung im Fallbeispiel der GDU SAP Services beruht sowohl auf der Prognose als auch auf vorliegenden Kundenverträgen für IT-Services (Ebert, 2009, S. 47 ff.). Aufgrund unterschiedlicher Eintrittswahrscheinlichkeiten für die Verträge wird mit verschiedenen Status gearbeitet (z. B. „erwarteter Vertrag“" oder ,abgeschlossener Vertrag“). Eine übliche Planungsannahme für die Vertragslaufzeit für die SAP-Services sind drei Jahre. Die Berücksichtigung bestehender IT-Service-Instanzen erfolgt mittels einer Kennzeichnung des Bedarfs mit „Neukunde“ oder „Bestandskunde“. Aus den IT-Service-Bedarfen wird der Bedarf an Betriebsmitteltypen abgeleitet. Die Auswahl eines geeigneten Betriebsmittels wird u.a. nach kapazitiven Gesichtspunkten getroffen und umfasst die Zuordnung eines konkreten Produktionsstandorts (Rechenzentrums).

\subsection{Mengenplanung}

Die Bedarfsinformationen für Betriebsmitteltypen gehen unmittelbar in die Mengenplanung ein. Analog zur industriellen Fertigung findet für Komponenten von Betriebsmitteltypen eine Brutto-/Netto-Bedarfsrechnung unter Berücksichtigung der Lagerbestände (inkl. Stücklistenauflösung), die Losbildung und schließlich die Vorlaufverschiebung statt (Zäpfel, 1996, S. 126ff.). Andere Komponenten, deren Bedarf nicht abgeleitet werden kann oder soll, werden verbrauchsorientiert auf Basis von Vergangenheitswerten disponiert. Für die Unterscheidung zwischen bedarfs- und verbrauchsorientiert disponierbaren Komponenten kann z. B. eine ABC-Analyse genutzt werden (Kurbel, 2005, S. 112). Das Ergebnis der Mengenplanung sind die Fertigungs- bzw. Beschaffungsaufträge, die Mengen je Periode enthalten (Kurbel, 2005, S. 134).

Im Fallbeispiel sind die zentralen Planungsgrößen der Mengenplanung zur frühzeitigen Disposition der notwendigen Ressourcen die Leistungsmengen in SAPS, die daraus abgeleiteten Slices sowie der Speicherbedarf in GByte (Ebert, 2009, S. 47 ff.). Hierbei werden bestehende „Lagerbestände“ in den Ressourcenpools berücksichtigt und ggf. die Beschaffung zusätzlicher Ressourcen ausgelöst. Im Falle der Server-Kapazitäten erfolgt die Bereitstellung in Losen (,ServerPaletten"). Dabei wird mit einem Sicherheitsbestand an vorzuhaltenden Kapazitäten gearbeitet. Am Ende der Mengenplanung werden für die beteiligten Arbeitsplätze Aufträge generiert, die den Fertigungsaufträgen gleichzusetzen sind.

\subsection{Termin- und Kapazitätsplanung}

Im Anschluss an die Mengenplanung wird die Terminplanung durchgeführt. In der Bereitstellung von Betriebsmitteln werden Lieferfristen über SLA fixiert. Da- 
her hat die Liefertreue Vorrang vor der Kapazitätsauslastung und die Kapazitäten werden möglichst flexibilisiert, um auf Bedarfsschwankungen reagieren zu können. Die zeitliche Terminierung von Fertigungsaufträgen für Komponenten wird zunächst ohne Berücksichtigung von Kapazitätsrestriktionen mit dem Ziel der fristgerechten Einplanung vorgenommen. Erst in einem zweiten Schritt werden Kapazitätsrestriktionen berücksichtigt, die zu einer zeitlichen Verschiebung führen können (Kurbel, 2005, S. 139 ff.). Die Kapazitätsplanung in der Bereitstellung hat primär die Anpassung der Kapazitäten und nur sekundär die zeitliche Verschiebung der Aufträge zum Ziel. Im Mittelpunkt der Betrachtung stehen - wie in der industriellen Fertigung - jedoch nur solche Arbeitsplätze, die potenzielle Engpässe darstellen. Der Dispositionsspielraum für Fertigungsaufträge liegt nur innerhalb des vertraglich vereinbarten Rahmens.

Das Fallbeispiel zeigt einen Fokus auf die Berücksichtigung erwarteter Vorlaufzeiten für die Arbeitsgänge auf, für die zum Teil Planvorgaben existieren (Ebert, 2009, S. 47 ff.). Einen besonderen Engpass kann hierbei die WANAnbindung des Kundenstandorts an die Rechenzentren der T-Systems darstellen. Diese kann mehrere Wochen dauern, was dem Vielfachen der Zeit für die Bereitstellung innerhalb des Rechenzentrums entspricht.

\subsection{Auftragsveranlassung und-überwachung}

Abschließend erfolgt die Auftragsveranlassung und -überwachung zur Veranlassung der Fertigungsaufträge, der Überwachung ihrer Ausführung und der Steuerung bei Planabweichungen. Hierbei werden Betriebsmittel bereitgestellt und zu IT-Service-Instanzen zugeordnet. Von besonderer Bedeutung ist die Einhaltung der vereinbarten Fristen der SLA. Zu diesem Zweck sollte eine Feinterminierung auf Basis von Prioritäten (z. B. „Chefauftrag“) vorgenommen werden (Kurbel, 2005, S. 167 ff.).

In der Fallstudie stellt während der Veranlassung, Überwachung und Steuerung der Aufträge insbesondere die Koordination verschiedener involvierter Bereiche im Gesamtprozess eine Herausforderung dar (Ebert, 2009, S. 47 ff.). Die Steuerung wird im Hinblick auf die geplanten Durchlaufzeiten und SLA-Vorgaben vorgenommen.

\section{Möglichkeiten und Grenzen des PPS-Einsatzes}

Die vorangegangenen Erläuterungen und das begleitende Fallbeispiel zeigen auf, dass im Wandel vom projektbasierten zum kundenorientierten, standardisierten IT-Serviceangebot PPS-Systeme für das IT-Service-Management zur wiederkehrenden, effizienten Produktion genutzt werden können. Da die Bereitstellung durch den Kunden ausgelöst wird, scheinen MRP II-basierte PPS-Systeme besser 
geeignet als z. B. Ansätze wie Just-in-Time oder Kaizen (Schönsleben, 2000). In der Vergangenheit konnte man durch PPS-Systeme folgende Vorteile nachweisen, wie die Verringerung der Fertigungskosten, die Senkung der Lagerbestände und die Verkürzung von Durchlaufzeiten (Matsui and Sato, 2002).

Durch den Einsatz von PPS-Systemen zur Bereitstellung von IT-Services sind diese Vorteile ebenfalls denkbar. Die Senkung der Lagerbestände stellt auch für die Provider eine zentrale Herausforderung dar. Aufgrund des hohen Kostendrucks werden Sicherheitsbestände von Systemkapazitäten sehr wahrscheinlich hinterfragt werden müssen. Im IT-Sektor verschärft sich die Problematik insbesondere durch die kurzen Technologielebenszyklen und die damit verbundene Alterung der eingesetzten Komponenten. Desgleichen ist auch die Verkürzung der Durchlaufzeiten der Bereitstellung für die IT-Organisationen ein wichtiges Ziel, um die Einhaltung der SLA sicherzustellen. Der Einsatz von PPS-Systemen verspricht jedoch nicht nur Produktivitätsvorteile. Auch indirekte Nutzenpotenziale durch Verwendung der Daten der PPS für andere Unternehmensbereiche (wie z. B. das Controlling) sind denkbar.

Das dargestellte Fallbeispiel macht die Voraussetzungen für den PPS-Einsatz deutlich. Die Nutzung von PPS bei sehr kundenindividuellen „Professional Services“ ist nicht sinnvoll. Stattdessen sind ein hoher Standardisierungsgrad von ITServices, Systemen und Prozessen z. B. zur Abbildung der IT-Services im SelfService-Portal und der Daten mittels Stücklisten, Arbeitsplänen etc. notwendig. Auch muss die Anwendbarkeit einzelner PPS-Aufgaben differenziert betrachtet werden. Z. B. verlagert sich aufgrund der zunehmenden Automatisierung der Bereitstellungsprozesse der Aufgabenschwerpunkt der PPS von der kurzfristigen Produktionssteuerung zur mittelfristigen Mengenplanung - wie dies bei flexiblen Fertigungssystemen der Fall war (Tempelmeier and Kuhn, 1993).

Jedoch sind dem Einsatz der heutigen PPS-Systeme auch Grenzen gesetzt. Eine vertragsorientierte Programmplanung wird nach Kenntnisstand der Autoren ebenso wenig unterstützt wie die Berücksichtigung des Bestands an IT-ServiceInstanzen und Betriebsmitteln. Hierzu sind Erweiterungen erforderlich, die in einem Praxisprojekt der Autoren erfolgreich konzeptioniert und in einem SoftwarePrototyp implementiert werden konnten (Ebert, 2009, S. 164 ff.). Eine weitere Schwäche der PPS-Systeme betrifft die Kapazitäten. Die IT-Organisationen arbeiten häufig mit flexiblen Personalkapazitäten, weswegen die Planungsfunktionen von PPS-Systemen zu unflexibel sind, da sie von starren Kapazitäten ausgehen. Eine weitere Schwäche der PPS-Systeme betrifft die sehr kurzfristige Steuerung. $\mathrm{Zu}$ diesem Zweck werden in der Praxis ergänzend zu den PPS-Systemen Manufacturing-Execution-Systems (MES) eingesetzt, die bei Providern den ITILkonformen IT-Service-Management-Systemen gleichzusetzen sind. 


\section{Literatur}

Capgemini (2008) Studie IT-Trends 2008. Berlin, CapGemini.

Corsten H \& Gössinger R (2007) Dienstleistungsmanagement, München, R. Oldenbourg Verlag.

Ebert N (2009) Produktionsplanung und -steuerung bei IT-Dienstleistern. St.Gallen, Universität St.Gallen.

Ebert N \& Vogedes A (2008) Dynamic Services for SAP Solutions der T-Systems Enterprise Services GmbH. St. Gallen, Universität St. Gallen - Institut für Wirtschaftsinformatik http://www.alexandria.unisg.ch/Publikationen/49181 (Stand: 2009-1217)..

Ebert N, Vogedes A \& Uebernickel F (2009) Produktionsplanung und -steuerung der IT-Service-Provisionierung. 9. Internationale Tagung Wirtschaftsinformatik. Wien.

Garschhammer M, Hauck R, Hegering H G, Kempter B, Radisic I, Rolle H, Schmidt H, Langer M \& Nerb M (2001a) Towards generic Service Management Concepts - A Service Model Based Approach. 7th IFIP/IEEE International Symposium on Integrated Network Management.

Garschhammer M, Hauck R, Kempter B, Radisic I, Roelle H \& Schmidt H (2001b) The MNM Service Model - Refined Views on Generic Service Management. Journal of Communications and Networks, 3, 297-306.

Gartner (2008) IT Key Metrics Data 2009: Executive Summary. Stamford, Gartner.

Grawe T \& Fähnrich K-P (2008) Service Engineering bei IT-Dienstleistern. In Fähnrich K-P \& van Husen C (Eds.) Entwicklung IT-basierter Dienstleistungen. Heidelberg, Physica.

Hackstein R (1989) Produktionsplanung und-steuerung (PPS). Ein Handbuch für die Betriebspraxis, VDI-Verl.

Kurbel K (2005) Produktionsplanung und Produktionssteuerung im Enterprise Resource Planning und Supply Chain Management, München, Oldenbourg.

Lebrecht A (1991) Die Anwendung des CIM-Konzeptes auf den DV-Betrieb. Organisation und Betrieb von Informationssystemen, 9. GI-Fachgespräch über Rechenzentren. Springer-Verlag.

Matsui Y \& Sato O (2002) An International Comparison Study on Benefits of Production Information Systems. International Journal of Operations and Quantitative Management 8, 191-214.

McKinsey \& Company (2008) Revolutionizing Data Center Efficiency.

Mertens P (2007) Integrierte Informationsverarbeitung 1: Operative Systeme in der Industrie. , Wiesbaden, Gabler. 
Mißbach M \& Stelzel J (2005) Adaptive Hardware-Infrastrukturen für SAP, Galileo Press.

OGC (2007) ITIL - Service Operation, Norwich, The Stationary Office.

Osterburg S, Pinnow A, Rautenstrauch C \& Winter M (2009) Neue ComputingGrundlagen für das Rechenzentrum. Informatik-Spektrum, 32, 118-126.

Schönsleben P (2000) Varying concepts of planning and control in enterprise logistics. Production Planning \& Control, 11, 2-6.

Tempelmeier H \& Kuhn H (1993) Flexible Fertigungssysteme: Entscheidungsunterstützung für Konfiguration und Betrieb, Berlin, Springer.

Zäpfel G (1982) Produktionswirtschaft - Operatives Produktions-Management, Berlin/New York, de Gruyter.

Zäpfel G (1996) Grundzüge des Produktions- und Logistikmanagement, Berlin/New York, de Gruyter.

Zarnekow R (2007) Produktionsmanagement von IT-Dienstleistungen. Grundlagen, Aufgaben und Prozesse, Berlin, Springer Verlag. 